\title{
Auto-exame das mamas como fator de prevenção ao câncer: uma abordagem com estudantes de uma escola pública da cidade de São Francisco do Conde - Bahia
}

\author{
Michele Santana Pacheco de Almeida
}

Estudante de Pós-Graduação em Especialização em Gestão de Saúde. Programa Nacional de Administração Pública, Universidade Aberta do Brasil. Universidade da Integração Internacional da Lusofonia AfroBrasileira. Campus de São Francisco do Conde, Bahia, Brasil.

\section{Vânia de Santana Silva}

Estudante de Pós-Graduação em Especialização em Gestão de Saúde. Programa Nacional de Administração Pública, Universidade Aberta do Brasil. Universidade da Integração Internacional da Lusofonia AfroBrasileira. Campus de São Francisco do Conde, Bahia, Brasil.

\section{Itaciara de Oliveira do Carmo da Silva}

Estudante de Pós-Graduação em Especialização em Gestão de Saúde. Programa Nacional de Administração Pública, Universidade Aberta do Brasil. Universidade da Integração Internacional da Lusofonia AfroBrasileira. Campus de São Francisco do Conde, Bahia, Brasil.

\section{Deivid Silva de Araújo Esquivel}

Estudante de Pós-Graduação em Especialização em Gestão de Saúde. Programa Nacional de Administração Pública, Universidade Aberta do Brasil. Universidade da Integração Internacional da Lusofonia AfroBrasileira. Campus de São Francisco do Conde, Bahia, Brasil.

ALMEIDA, Michele Santana Pacheco de; SILVA, Vânia de Santana; SILVA, Itaciara de Oliveira do Carmo da Silva; ESQUIVEL, Deivid Silva de Araújo; SANTOS, Ana Paula do Carmo; SILVEIRA, Helson Freitas da; FREITAS, Ana Paula Fragoso de; JÚNIOR; Howard Lopes Ribeiro. Auto-exame das mamas como fator de prevenção ao câncer: uma abordagem com estudantes de uma escola pública da cidade de São Francisco do Conde - Bahia. Revista Intertox-EcoAdvisor de Toxicologia Risco Ambiental e Sociedade, v. 8, n. 1, p. 100-112, fev. 2015. 


\section{Ana Paula do Carmo Santos}

Estudante de Pós-Graduação em Especialização em Gestão de Saúde. Programa Nacional de Administração Pública, Universidade Aberta do Brasil. Universidade da Integração Internacional da Lusofonia AfroBrasileira. Campus de São Francisco do Conde, Bahia, Brasil.

\section{Helson Freitas da Silveira}

Programa de Pós-Graduação em Ciências Morfofuncionais. Universidade Federal do Ceará, Fortaleza, Ceará, Brasil.

\section{Ana Paula Fragoso de Freitas}

Programa de Pós-Graduação em Ciências Médicas. Universidade Federal do Ceará, Fortaleza, Ceará, Brasil.

\section{Howard Lopes Ribeiro Júnior}

Professor-Pesquisador II da Especialização em Gestão de Saúde. Programa Nacional de Formação em Administração Pública, Universidade Aberta do Brasil. Universidade da Integração Internacional da Lusofonia Afro-Brasileira, Campus de São Francisco do Conde, Bahia, Brasil.

E-mail: howard@unilab.edu.br

ALMEIDA, Michele Santana Pacheco de; SILVA, Vânia de Santana; SILVA, Itaciara de Oliveira do Carmo da Silva; ESQUIVEL, Deivid Silva de Araújo; SANTOS, Ana Paula do Carmo; SILVEIRA, Helson Freitas da; FREITAS, Ana Paula Fragoso de; JÚNIOR; Howard Lopes Ribeiro. Auto-exame das mamas como fator de prevenção ao câncer: uma abordagem com estudantes de uma escola pública da cidade de São Francisco do Conde - Bahia. Revista Intertox-EcoAdvisor de Toxicologia Risco Ambiental e Sociedade, v. 8, n. 1, p. 100-112, fev. 2015. 


\section{凡}

\section{RESUMO}

O presente trabalho buscou avaliar o conhecimento que as estudantes da Educação de Jovens e Adultos no Ensino Médio, de uma escola pública do município de São Francisco do Conde, na Bahia, apresentam sobre conceitos e importância do exame das mamas como fator preventivo ao câncer de mama precoce. A pesquisa se desenvolveu mediante um estudo exploratório do tipo descritivo, quantitativo com base em um questionário avaliativo, aplicado a uma população constituída por mulheres com a faixa etária entre 18 e 70 anos. A coleta de dados foi realizada com 124 estudantes que se disponibilizaram em responder um questionário com perguntas abertas e fechadas sobre a realização do exame das mamas e o seu impacto na prevenção do câncer de mama. Os principais resultados indicaram que: houve uma predominância de estudantes na faixa de idade de 20 a 49 anos, autodenominadas negras, com estado civil solteiro e sem filhos. Adicionalmente, avaliamos que predominou mulheres que conhecem o exame das mamas, mas não o realiza, e que frequentam o médico ginecologista ou clinico geral de 2 a 3 vezes ao ano, porém o profissional de saúde não realiza o exame das mamas na maioria dos casos. A partir do presente estudo foi possível ser constatado que as informações e orientações quanto às ações de prevenção ao câncer de mama na saúde pública do referido munícipio tem sido pouco efetivas, necessitando, portanto, de uma intensificação das atividades desenvolvidas pelos profissionais de saúde que atuam nas atividades relacionadas à saúde da mulher, promovendo um aumento na conscientização da importância da realização do exame das mamas periodicamente.

Palavras - chave: Câncer de mama; Conhecimento sobre câncer de mama; Saúde da Mulher.

\section{ABSTRACT}

This study aimed to evaluate the knowledge that the students of the Youth and Adult Education in High School, a public school in São Francisco do Conde, Bahia, present on concepts and importance of breast examination as a preventive factor for cancer early breast. The research was developed through an exploratory study of descriptive, quantitative type based on an evaluation questionnaire administered to a population made up of women aged between 18 and 70 years. Data collection was performed with 124 students who agreed to answer a questionnaire with open and closed questions on the examination of the breasts and their impact on the

ALMEIDA, Michele Santana Pacheco de; SILVA, Vânia de Santana; SILVA, Itaciara de Oliveira do Carmo da Silva; ESQUIVEL, Deivid Silva de Araújo; SANTOS, Ana Paula do Carmo; SILVEIRA, Helson Freitas da; FREITAS, Ana Paula Fragoso de; JÚNIOR; Howard Lopes Ribeiro. Auto-exame das mamas como fator de prevenção ao câncer: uma abordagem com estudantes de uma escola pública da cidade de São Francisco do Conde - Bahia. Revista Intertox-EcoAdvisor de Toxicologia Risco Ambiental e Sociedade, v. 8, n. 1, p. 100-112, fev. 2015. 


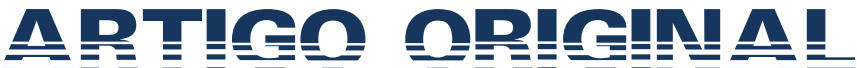

prevention of breast cancer. The main results showed that: there was a predominance of students in the age group 20-49 years old, self-styled black, with unmarried and childless. Additionally, we evaluated that prevailed women who know the breast exam, but do not realize it, and attending the gynecologist or general practitioner doctor 2-3 times a year, but the provider does not perform breast examination in most cases. From the present study it was possible to be seen that the information and guidance on the actions to prevent breast cancer in public health of this municipality has been ineffective, requiring therefore an intensification of the activities developed by health professionals working in activities related to women's health, promoting an increase in awareness of the importance of the examination of the breasts regularly.

Keywords: Breast cancer; Knowledge about breast cancer; Women's Health

\section{INTRODUÇÃO}

O Câncer de Mama consiste em uma neoplasia maligna, hereditária ou não, desenvolvida no tecido mamária e correspondendo a principal neoplasia no mundo que leva ao óbito mulheres com idade superior a 50 anos (MARTINS 2009; BORGES, 2010; BIZERRA, 2013). O Instituto Nacional do Câncer (INCA) estima que o quantitativo de novos casos de câncer de mama no Brasil, em 2014, será de 57.120 (SILVA; FRANCO; MARQUESINCA, 2005; INCA, 2014) com um risco estimado de 56,09 casos a cada 100 mil mulheres. Já no estado da Bahia, de acordo com o INCA, foram previstos 2.560 casos novos de câncer de mama no estado da Bahia até o final de 2014, sendo 980 destes em Salvador (SILVA; FRANCO; MARQUESINCA, 2005; CUNHA, 2009, p. 3; INCA, 2014).

Em relação aos fatores de risco que podem favorecer a minimização dos casos de câncer de mama, podem ser destacados os relacionados à idade, especialmente em mulheres acima dos 40 anos, a terapia de reposição hormonal e o histórico de câncer na família. Fatores comportamentais ALMEIDA, Michele Santana Pacheco de; SILVA, Vânia de Santana; SILVA, Itaciara de Oliveira do Carmo da Silva; ESQUIVEL, Deivid Silva de Araújo; SANTOS, Ana Paula do Carmo; SILVEIRA, Helson Freitas da; FREITAS, Ana Paula Fragoso de; JÚNIOR; Howard Lopes Ribeiro. Auto-exame das mamas como fator de prevenção ao câncer: uma abordagem com estudantes de uma escola pública da cidade de São Francisco do Conde - Bahia. Revista Intertox-EcoAdvisor de Toxicologia Risco Ambiental e Sociedade, v. 8, n. 1, p. 100-112, fev. 2015. 


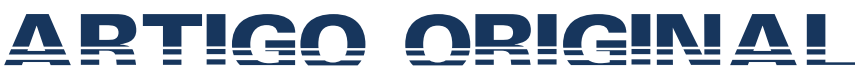

também no acometimento do câncer de mama, tais como a obesidade, o tabagismo e o uso do álcool (INCA, 2014).

Segundo Silva et al (2009), o exame da mama consiste em um exame físico simples, podendo ser realizado pela própria mulher (autoexame) ou por profissional da saúde especializado, sem causas de dor, gratuito e essencial para a deteç̧ão precoce do câncer de mama, prolongando a sobrevida da paciente (SILVA et al, 2009; INCA, 2014). É fundamental, também para o conhecimento mais aprofundado pela mulher das próprias mamas de forma a familiarizar-se com a forma, tamanho, aspecto da pele e do mamilo, o que vai facilitar precocemente, a deteç̧ão de anormalidades possibilitando um bom prognóstico, podendo evitar a mutilação da mama.

Baseado neste contexto, o presente trabalho é resultado de uma pesquisa pioneira desenvolvida no município de São Francisco do Conde BA, buscando avaliar o conhecimento de mulheres estudantes do Ensino Médio de um colégio da rede pública de ensino sobre o câncer de mama e a realização do autoexame, seja pela própria mulher ou pelo médico assistencialista da rede pública de saúde, frente à conscientização e minimização da ocorrência desta neoplasia no referido município.

\section{MÉTODOS}

A presente pesquisa trata-se de um estudo exploratório do tipo descritivo, quantitativo, utilizando-se de dados primários obtidos a partir de questionários avaliativos aplicados a estudantes matriculadas no curso de Educação de Jovens e Adultos do Ensino Médio em um colégio público estadual localizado no município de São Francisco do Conde - Bahia, buscando a obtenção de informações sobre o conhecimento e a realização do autoexame como fator de proteção ao câncer de mama precoce.

ALMEIDA, Michele Santana Pacheco de; SILVA, Vânia de Santana; SILVA, Itaciara de Oliveira do Carmo da Silva; ESQUIVEL, Deivid Silva de Araújo; SANTOS, Ana Paula do Carmo; SILVEIRA, Helson Freitas da; FREITAS, Ana Paula Fragoso de; JÚNIOR; Howard Lopes Ribeiro. Auto-exame das mamas como fator de prevenção ao câncer: uma abordagem com estudantes de uma escola pública da cidade de São Francisco do Conde - Bahia. Revista Intertox-EcoAdvisor de Toxicologia Risco Ambiental e Sociedade, v. 8, n. 1, p. 100-112, fev. 2015. 


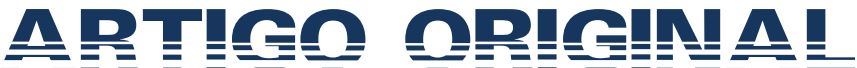

Os dados foram coletados a partir das respostas de 124 mulheres estudantes, de um total de 368 alunos matriculados na referida unidade de ensino. A análise dos dados possibilitou obter descrições sobre aspectos sócio demográficos das estudantes, com as variáveis: faixa etária, naturalidade, etnia, estado civil e número de filhos, seguida da descrição das variáveis associadas a realização do autoexame como fator de prevenção ao acometimento do câncer de mama tais como frequência de ida ao clínico geral e ao ginecologista e sobre o conhecimento e a realização do autoexame das mama tanto pela estudante quanto pelo médico.

O presente estudo foi submetido e aprovado pelo Comitê de Ética em Pesquisa com Seres Humanos da Universidade da Integração Internacional da Lusofonia Afro-Brasileira sob número de protocolo: 566.468. Os dados obtidos a partir das análises das variáveis contidas nos questionários foram tabulados e analisados através da estatística analítico-descritiva, com auxílio do software Microsoft Office Excel 2010.

\section{RESULTADOS}

Do total de 124 estudantes avaliadas, observamos uma predominância de estudantes na faixa etária entre 20 a 49 anos de idade (68,0\%), seguida de estudantes na faixa etária de 18 a 19 anos (29,4\%); $54,5 \%$ e $37,4 \%$ das mulheres considerando-se negras e pardas, respectivamente; $78,0 \%$ das mulheres apresentando estado civil de solteiras e 65,3\% das entrevistadas declarando-se não possuírem filhos (Tabela 1).

Com relação as variáveis associadas ao autoexame da mama como fator preventivo ao câncer de mama, avaliamos que 57,8\% (70/121) das estudantes afirmam que conhecem o autoexame das mamas, mas não o realiza em 67,2\% (80/119) dos casos (Tabela 2). Adicionalmente, observamos

ALMEIDA, Michele Santana Pacheco de; SILVA, Vânia de Santana; SILVA, Itaciara de Oliveira do Carmo da Silva; ESQUIVEL, Deivid Silva de Araújo; SANTOS, Ana Paula do Carmo; SILVEIRA, Helson Freitas da; FREITAS, Ana Paula Fragoso de; JÚNIOR; Howard Lopes Ribeiro. Auto-exame das mamas como fator de prevenção ao câncer: uma abordagem com estudantes de uma escola pública da cidade de São Francisco do Conde - Bahia. Revista Intertox-EcoAdvisor de Toxicologia Risco Ambiental e Sociedade, v. 8, n. 1, p. 100-112, fev. 2015. 


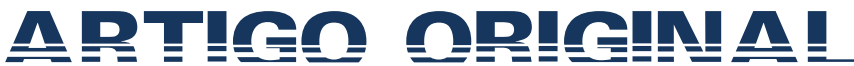

que $47,9 \% \quad(57 / 119)$ e $47,0 \% \quad(55 / 117)$ das mulheres frequentam, respectivamente, o clínico geral e o ginecologista entre 2 a 3 vezes ao ano (Tabela 2). Ainda referente à ida da estudante ao ginecologista, observamos que 58,3\% (70/120) das mulheres informaram que o ginecologista não examina suas mamas no momento da consulta.

Tabela 1: Descrição dos aspectos sócio demográficos.

\begin{tabular}{lcc}
\hline \multicolumn{1}{c}{ Variáveis } & $\mathbf{N}^{\mathbf{0}}$ de Pacientes $\mathbf{( N = 1 2 4 )}$ & $\mathbf{\%}$ \\
\hline Faixa Etária*, ** & & \\
18 a 19 anos & 35 & 29,4 \\
20 a 49 anos & 81 & 68,0 \\
50 anos ou mais & 3 & 2,5 \\
Naturalidade & & \\
São Francisco do Conde & 38 & 30,6 \\
Outras localidades & 86 & 69,4 \\
Etnia* & & \\
Branca & 4 & 3,3 \\
Parda & 46 & 37,4 \\
Negra & 67 & 54,5 \\
Amarela & 2 & 1,6 \\
Indígena & 4 & 3,3 \\
Estado Civil* & & \\
Solteira & 96 & 78,0 \\
Casada & 21 & 17,1 \\
Separada/Divorciada/Desquitada & 3 & 2,4 \\
Viúva & 3 & 2,4 \\
Possui Filhos* & & \\
Sim & 43 & 34,7 \\
Não & 81 & 65,3 \\
\hline
\end{tabular}

* 1 (um) ou mais participantes não responderam ao questionamento.

ALMEIDA, Michele Santana Pacheco de; SILVA, Vânia de Santana; SILVA, Itaciara de Oliveira do Carmo da Silva; ESQUIVEL, Deivid Silva de Araújo; SANTOS, Ana Paula do Carmo; SILVEIRA, Helson Freitas da; FREITAS, Ana Paula Fragoso de; JÚNIOR; Howard Lopes Ribeiro. Auto-exame das mamas como fator de prevenção ao câncer: uma abordagem com estudantes de uma escola pública da cidade de São Francisco do Conde - Bahia. Revista Intertox-EcoAdvisor de Toxicologia Risco Ambiental e Sociedade, v. 8, n. 1, p. 100-112, fev. 2015. 


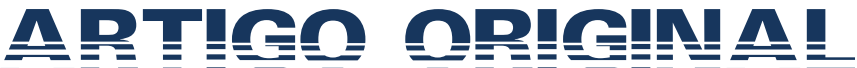

Tabela 2: Descrição das variáveis associadas ao autoexame da mama como fator preventivo ao câncer de mama.

\begin{tabular}{lcc}
\hline \multicolumn{1}{c}{ Variáveis } & $\mathbf{N}^{\mathbf{0}}$ de Pacientes $\mathbf{~ N = 1 2 4 )}$ & $\mathbf{\%}$ \\
\hline Frequência de ida ao clínico geral* & 33 & 27,7 \\
$0-1$ vez por ano & 57 & 47,9 \\
2-3 vezes por ano & 29 & 24,4 \\
4 ou mais vezes por ano & & \\
Frequência de ida ao ginecologista* & 47 & 40,2 \\
$0-1$ vez por ano & 55 & 47,0 \\
2-3 vezes por ano & 15 & 12,8 \\
4 ou mais vezes por ano & & \\
Conhece o autoexame das mamas* & 70 & 57,8 \\
Sim & 51 & 42,2 \\
Não & & \\
Realiza o autoexame das mamas* & 13 & 10,9 \\
Diariamente & 14 & 11,8 \\
Ocasionalmente & 12 & 10,1 \\
Raramente & 80 & 67,2 \\
Não realiza & & \\
Realização do exame da mama pelo ginecologista* & 50 & 41,7 \\
Sim & 70 & 58,3 \\
Não & & \\
\hline
\end{tabular}

* 1 (um) ou mais participantes não responderam ao questionamento.

Quanto à caracterização das variáveis relacionadas às campanhas municipais de saúde da cidade de São Francisco do Conde frente à promoção da prevenção ao câncer de mama, identificamos que as estudantes reconhecem $(84,4 \%, 103 / 122)$ e veem como importante $(98,4 \%, 122 / 124)$ a realização das campanhas de saúde promovidas pelo município em questão (Tabela 3). No entanto, 59,1\% (55/93) das estudantes informam que raramente participam das campanhas. Adicionalmente, 59,1\% (100/124) das mulheres avaliadas informam que falta orientação nos postos de saúde frente à prevenção ao câncer de mama (Tabela 3).

Tabela 3: Descrição das variáveis relacionadas às campanhas municipais de prevenção ao Câncer de Mama.

ALMEIDA, Michele Santana Pacheco de; SILVA, Vânia de Santana; SILVA, Itaciara de Oliveira do Carmo da Silva; ESQUIVEL, Deivid Silva de Araújo; SANTOS, Ana Paula do Carmo; SILVEIRA, Helson Freitas da; FREITAS, Ana Paula Fragoso de; JÚNIOR; Howard Lopes Ribeiro. Auto-exame das mamas como fator de prevenção ao câncer: uma abordagem com estudantes de uma escola pública da cidade de São Francisco do Conde - Bahia. Revista Intertox-EcoAdvisor de Toxicologia Risco Ambiental e Sociedade, v. 8, n. 1, p. 100-112, fev. 2015. 


\begin{tabular}{|c|c|c|}
\hline Variáveis & $\begin{array}{l}\mathrm{N}^{\circ} \text { de Pacientes } \\
(\mathrm{N}=124)\end{array}$ & $\%$ \\
\hline \multicolumn{3}{|l|}{$\begin{array}{l}\text { As estudantes acham importante a realização de campanhas frente à prevenção ao } \\
\text { câncer de mama? }\end{array}$} \\
\hline $\operatorname{Sim}$ & 122 & 98,4 \\
\hline Não & 2 & 1,6 \\
\hline \multicolumn{3}{|l|}{$\begin{array}{l}\text { As estudantes veem que a SESA municipal realiza campanhas frente à prevenção ao } \\
\text { câncer de mama?* }\end{array}$} \\
\hline $\operatorname{Sim}$ & 103 & 84,4 \\
\hline Não & 19 & 15,6 \\
\hline \multicolumn{3}{|l|}{$\begin{array}{l}\text { As estudantes participam das campanhas frente à prevenção ao câncer de mama } \\
\text { promoyidaspela SESA?* }\end{array}$} \\
\hline Sempre & 16 & 17,2 \\
\hline Ocasionalmente & 22 & 23,7 \\
\hline Raramente & 55 & 59,1 \\
\hline \multicolumn{3}{|l|}{$\begin{array}{l}\text { As estudantes acham que falta orientação nos postos de saúde frente a prevenção ao } \\
\text { câncer de mama? }\end{array}$} \\
\hline $\operatorname{Sim}$ & 100 & 80,6 \\
\hline Não & 24 & 19,4 \\
\hline
\end{tabular}

* 1 (um) ou mais participantes não responderam ao questionamento.

\section{DISCUSSÃO}

Nesse estudo, em relação às variáveis sociodemográficas (faixa etária, etnia, estado civil), constatamos que a maioria das estudantes é de cor negra e solteira. Em relação à variável faixa etária, 40,3\% das estudantes apresentou predominância de 20 a 29 anos, faixa de idade em que não é frequente a ocorrência do câncer de mama em mulheres, pois estudos comprovam que mulheres com menos de 30 anos não apresentam altas incidências da doença (TESSARO, 1999). No entanto, é sempre relevante considerar a importância da realização de exames clínicos na mama como prática de prevenção e detecção precoce ao câncer de mama.

Em relação à cor da pele, 54,5\% das alunas classificaram-se negras. Em relação a essa variável, a prevalência de surgimento de neoplasias de mama são maiores nas mulheres de pele branca, porém o diagnóstico tardio é realizado mais frequentemente na população feminina afrodescendente (BAIRROS; MENEGHEL; OLINTO, 2008). Segundo estudos, mulheres ALMEIDA, Michele Santana Pacheco de; SILVA, Vânia de Santana; SILVA, Itaciara de Oliveira do Carmo da Silva; ESQUIVEL, Deivid Silva de Araújo; SANTOS, Ana Paula do Carmo; SILVEIRA, Helson Freitas da; FREITAS, Ana Paula Fragoso de; JÚNIOR; Howard Lopes Ribeiro. Auto-exame das mamas como fator de prevenção ao câncer: uma abordagem com estudantes de uma escola pública da cidade de São Francisco do Conde - Bahia. Revista Intertox-EcoAdvisor de Toxicologia Risco Ambiental e Sociedade, v. 8, n. 1, p. 100-112, fev. 2015. 


\section{凡}

negras fazem menos os exames essenciais para detecção precoce do câncer de mama (BERGMAN, 2000). Com efeito, tal fato nos alerta para a necessidade de implantar políticas para melhorar o acesso aos serviços de saúde focados na população mais vulnerável no referido município.

Quanto ao estado civil das entrevistadas, observamos uma predominância de mulheres com estado civil solteiro (78,0\%). Estudos confirmam que o diagnóstico de câncer em mulheres solteiras traz grandes desafios, pois essas se encontram em fase reprodutiva, constituindo família e iniciando carreira profissional (GRIPPA et al., 2003). Sobretudo, observa-se que as mulheres casadas apresentam maior incidência da doença quando comparadas com as solteiras (BERGMANN, 2000). Das entrevistadas, cerca de 65,3\% responderam que não possuem filhos, e, sobre esse ponto, pesquisas indicam que o maior número de filhos e a maternidade podem ser fatores protetores ao câncer de mama (TESSARO, 1999).

Com relação aos fatores preventivos ao câncer de mama, 40,2\% das mulheres pesquisadas apresentaram uma baixa frequência na consulta ao ginecologista, apenas 0 -1 vez ao ano. A consulta ao médico é de fundamental importância na prevenção e detecção precoce do câncer mamário, conforme o INCA (2014). Sobre o uso de pílula anticoncepcional 23,7\% afirmaram usá-la, isso se constitui como fator de risco ao câncer de mama, segundo o INCA (2014).

Sobre o exame das mamas realizado pelo médico ginecologista, 58,3\% responderam que o médico em questão não realiza tal procedimento. Contudo, 57,8\% das entrevistadas afirmaram conhecerem o autoexame das mamas, enquanto que $67,2 \%$ desse total não realizam o autoexame das mamas com periodicidade. Segundo o INCA (2014), o exame das mamas realizado por profissionais de saúde (médicos e enfermeiros, por exemplo) é

ALMEIDA, Michele Santana Pacheco de; SILVA, Vânia de Santana; SILVA, Itaciara de Oliveira do Carmo da Silva; ESQUIVEL, Deivid Silva de Araújo; SANTOS, Ana Paula do Carmo; SILVEIRA, Helson Freitas da; FREITAS, Ana Paula Fragoso de; JÚNIOR; Howard Lopes Ribeiro. Auto-exame das mamas como fator de prevenção ao câncer: uma abordagem com estudantes de uma escola pública da cidade de São Francisco do Conde - Bahia. Revista Intertox-EcoAdvisor de Toxicologia Risco Ambiental e Sociedade, v. 8, n. 1, p. 100-112, fev. 2015. 


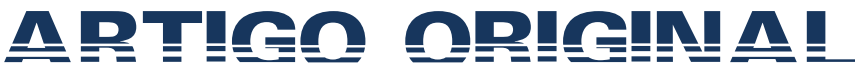

de extrema importância para deteç̧ão precoce do câncer de mama. Martins (2009, p. 22) considera que o exame clínico das mamas e a mamografia são de extrema relevância para a deteç̧ão precoce da doença, pois a redução da mortalidade está associada tanto pelo rastreamento quanto pelo tratamento adequado. Quando há um retardamento no diagnóstico as chances de sobrevida são reduzidas (INCA, 2014).

Em relação às campanhas municipais de prevenção ao câncer de mama, 98,4\% responderam que consideram importante a realização das campanhas, 84,4\% afirmaram que a Secretaria de Saúde realiza campanhas de prevenção, entretanto, $59,1 \%$ raramente participam de tais campanhas de prevenção ao câncer de mama. Sobre a orientação para prevenção ao câncer de mama nos postos de saúde, 80,6\% consideraram que falta orientação nos postos de saúde. Tais aspectos indicam que os postos de saúde do município precisam intensificar as atividades de prevenção ao câncer de mama para que as mulheres possam conhecer o próprio corpo, buscando cuidar da saúde, se atentando para a prevenção ao câncer de mama através de hábitos de vida saudáveis, a necessidade de frequentar o ginecologista com regularidade e da realização periódica do autoexame das mamas, como também o exame clínico nos hospitais pelo profissional médico.

\section{CONCLUSÕES}

A partir do presente estudo, estima-se que as estudantes do município de São Francisco do Conde necessitam de mais informações sobre prevenção ao câncer de mama, especialmente nos postos de atenção básica em saúde. A partir dessas informações, as mulheres sanfranciscanas poderão obter o conhecimento necessário para se prevenirem contra o câncer de mama que é uma das doenças que mais matam as mulheres no Brasil e no mundo,

ALMEIDA, Michele Santana Pacheco de; SILVA, Vânia de Santana; SILVA, Itaciara de Oliveira do Carmo da Silva; ESQUIVEL, Deivid Silva de Araújo; SANTOS, Ana Paula do Carmo; SILVEIRA, Helson Freitas da; FREITAS, Ana Paula Fragoso de; JÚNIOR; Howard Lopes Ribeiro. Auto-exame das mamas como fator de prevenção ao câncer: uma abordagem com estudantes de uma escola pública da cidade de São Francisco do Conde - Bahia. Revista Intertox-EcoAdvisor de Toxicologia Risco Ambiental e Sociedade, v. 8, n. 1, p. 100-112, fev. 2015. 


\section{凡}

segundo as pesquisas da OMS e do INCA. Estudos posteriores poderão ampliar a amostra de mulheres entrevistadas, buscando abranger um maior número de informações sobre o conhecimento das mulheres sanfranciscanas sobre o câncer de mama, possibilitando mais dados que visem melhorar as políticas públicas de saúde do município em questão.

\section{Agradecimentos}

Agradecemos o apoio da Sra. Valdinéia Ferreira Lima, diretora responsável pelo Colégio Estadual Matinho Salles Brasil, por permitir e disponibilizar o acesso da equipe executora da presente pesquisa para aplicar os questionários às estudantes foco desta pesquisa.

Este estudo foi apoiado e suportado pela Coordenação de Aperfeiçoamento de Pessoal de Nível Superior, CAPES, juntamente com a Universidade Aberta do Brasil - UAB.

\section{REFERÊNCIAS BIBLIOGRÁFICAS:}

BARROS, F. S.; MENEGHEL, S. N.; OLINTO, M. T. A. Citopatologia e exame de mama: desigualdade de acesso para mulheres negras no sul do Brasil. Epidemiol. Serv. Saúde, v.17, n.2, p.138-141, 2008.

BERGMANN, A. Prevalência de linfedema subsequente a tratamento cirúrgico para o câncer de mama. 2000. 132f. (Dissertação) (Mestrado) Escola Nacional de Saúde Pública, Rio de Janeiro, 2000.

BIZERRA, R.F.C; SANTOS, A. A. P.; SILVA, R. C.; MONTEIRO, E. K. R.; DOURADO, K. M. O. P.; SANTOS, J. A.; CERQUEIRA, G. S. Câncer de mama: prevenção e deteç̧ão precoce na atenção básica. Educación Física y Deportes, Revista Digital. Buenos Aires, Ano 17, nº 176, jan. 2013.

BORGES, R. Qualidade de vida de mulheres com câncer de mama e ginecológico mo município de Rio do Oeste segundo o olhar do WHOQOL BREF da OMS. Educación Física y Deportes, Revista Digital. Buenos Aires, Ano 15, no 149, out. 2010.

ALMEIDA, Michele Santana Pacheco de; SILVA, Vânia de Santana; SILVA, Itaciara de Oliveira do Carmo da Silva; ESQUIVEL, Deivid Silva de Araújo; SANTOS, Ana Paula do Carmo; SILVEIRA, Helson Freitas da; FREITAS, Ana Paula Fragoso de; JÚNIOR; Howard Lopes Ribeiro. Auto-exame das mamas como fator de prevenção ao câncer: uma abordagem com estudantes de uma escola pública da cidade de São Francisco do Conde - Bahia. Revista Intertox-EcoAdvisor de Toxicologia Risco Ambiental e Sociedade, v. 8, n. 1, p. 100-112, fev. 2015. 


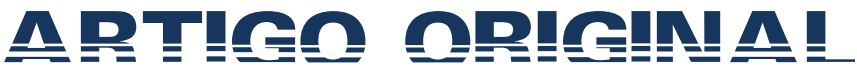

CUNHA, N. F. Políticas Públicas no Brasil com ênfase na prevenção do câncer de mama. 2009. 14f. (Monografia/Artigo) (Especialização em Vigilância Sanitária) - IFAR - Universidade Católica de Goiânia, Goiânia, 2009.

GRIPPA, C. G.; HALLAL, A. L. C.; DELLAGIUSTINA, A. R.; TRAEBERT, E. E.; GONDIN, G.; PEREIRA, C. Perfil clínico e epidemiológico do câncer de mama em mulheres jovens. Arquivos Catarinenses de Medicina, v. 32 , n. 3, p. 50-58, 2003.

INSTITUTO NACIONAL DO CÂNCER. Estimativa 2014. Incidência do Câncer no Brasil. Rio de Janeiro: INCA, 2014.

MARTINS, C. H. F. Ambiente de informações para apoio à decisão dos gestores do controle do câncer de mama. 2009. 120f. (Dissertação) (Mestrado Modalidade Profissional em Saúde Pública) - Escola Nacional de Saúde Pública Sérgio Arouca, Rio de Janeiro, 2009.

SILVA, N. C. B.; FRANCO, M. A. P.; MARQUES, S. L. Conhecimento de mulheres sobre câncer de mama e de colo do útero. Paidéia, v. 15, n. 32, p. 409-416, 2005.

SILVA, R. M. et al. Realização do autoexame das mamas por profissionais de enfermagem. Rev. esc. enferm. USP, São Paulo, v. 43, n. 4, Dec. 2009.

TESSARO, S. Epidemiologia do câncer de mama. In: Câncer de Mama (D. L. Basegio, org.), p. 1-11, Rio de Janeiro: Editora Revinter. 1999.

ALMEIDA, Michele Santana Pacheco de; SILVA, Vânia de Santana; SILVA, Itaciara de Oliveira do Carmo da Silva; ESQUIVEL, Deivid Silva de Araújo; SANTOS, Ana Paula do Carmo; SILVEIRA, Helson Freitas da; FREITAS, Ana Paula Fragoso de; JÚNIOR; Howard Lopes Ribeiro. Auto-exame das mamas como fator de prevenção ao câncer: uma abordagem com estudantes de uma escola pública da cidade de São Francisco do Conde - Bahia. Revista Intertox-EcoAdvisor de Toxicologia Risco Ambiental e Sociedade, v. 8, n. 1, p. 100-112, fev. 2015. 